\title{
A Quadratic Stability Solution for Switched Singular Linear Systems
}

\author{
Yu-Juan Yin, Jun Zhao, Georgi M. Dimirovski, and Tatyana D. Kolemisevska-Gugulovska
}

\begin{abstract}
Quadratic stability for a class of switched singular linear systems is investigated. The convex combination technique of standard switched linear systems is extended to switched singular systems and a sufficient condition for quadratic stability derived. A stabilizing switching law is also designed. For a switched singular system with two subsystems this appears a necessary and sufficient condition for quadratic stability. Also, the switching law realization is discussed relative to avoiding zeno behavior. An illustrative example is given to demonstrate the validity of these new results.
\end{abstract}

\section{INTRODUCTION}

$\mathrm{H}$ YBRID dynamical systems are complex systems possessing combined continuous-time and discrete-time dynamics, and even discrete-event dynamics [1-4]. Switched systems, as a typical and important class of hybrid systems, have drawn considerable attention during the recent years [4-6]. For switched systems appeared to find many practical applications in technological as well as social and economic systems inclusive in biological systems too. Also, as simplified models of hybrid systems, switched systems [7, 8] can often provide useful analysis tools and efficient design methods for more general hybrid systems.

On the other hand, it is known that singular systems appear in many engineering applications [9, 10]. Some examples are found in power systems, aerospace engineering, complex chemical processes, and therefore have been studied extensively [11]. It was not realized until

This research is supported in part by: the NSF of P.R. China China (Grant 60274009), the SRFDP (Grant 20020145007), and the NSF of Liaoning Province (Grant 20032020); Ministry of Education \& Science of the R. of Macedonia (Grant 143154); and Dogus University Fund for Science.

Yu-Juan Yin and Jun Zhao are with Northeastern University, School of Information Science and Engineering, Shenyang, 110004, P. R. of China (E-mails: yinyj64@ tom.com). Also, J. Zhao is with Australian National University, Research School of Information Science, Canberra, Australia (E-mail: jun.zhao@anu.edu.au).

T. D. Kolemisevska-Gugulovska and G.M. Dimirovski are with SS Cyril and Methodius University, FEIT-IASE, MK-1000 Skopje, Rep. of Macedonia. Also, G. M. Dimirovski is with Dogus University, AcibadeKadikoy, TR-34722, Istanbul, Rep. of Turkey (E-mail: gdimirovski@dogus.edu.tr ). recently that many singular systems also involve hybrid and switching features [13]. Usually, such systems are modeled either as singular systems without switching or as switched systems without singularity.

Recently considerable efforts have been made to study switched systems whose subsystems are either nonlinear [12] or unstable linear [13] or singular systems [14, 15]. Systems whose all subsystems are linear singular systems do represent a specific class of switched systems. For this class of systems only a few results have been available until very recently. Main obstacle reasons are the difficulties caused by the interaction between continuous dynamics, switching signal, and singularity. For instance, the impulse terms in singular subsystems and the reachable set of each subsystem as a sub-manifold in the sate space make the study of the existence and uniqueness of the solution much too complicated.

On the other hand, in practical applications of dynamic systems it is desirable to achieve quadratic stability property $[5,12,13]$ whenever possible. Study [5] has thoroughly explored the quadratic stabilizability of switched systems whereas [12] provided results on quadratic stability of nonlinear switched systems. Furthermore, studies [13-16] have provided the first useful results on techniques for stabilizing switched singular linear systems.

In the case of switched singular systems one important recent contribution was made in [15]. In this study, all subsystems are made to have the same algebraic constraints via state feedback, which converts the switched singular system into a standard switched system. On the other hand, in work [14], the derivatives of outputs are adopted in the feedback to normalize the singular subsystems, and thereafter the stability of switched systems is studied by LMI method. Apparently in both cases these normalization methods are very restrictive because of the need to satisfy very stringent conditions. Most such singular systems, however, can not be normalized.

Quite recently, works [16-19] proposed several new design results albeit via employing considerably more sophisticated control strategies. Fu and co-authors in [16-18] have derived their novel design results via applying $H_{\infty}$ [16] and robust $H_{\infty}[17,18]$ theory. On the other hand, Gao 
and $\mathrm{Wu}$ [19] proposed a novel LMI solution employing variable structure control theory.

The investigation of the control problem of concern by the present authors has begun with work [20] where the existence and uniqueness of solutions for switched singular linear systems were first explored. Yin and co-authors derived stability results for switched singular linear systems without [21] and with impulsive effects [22]. Subsequently in [23] they too gave the solution to $H_{\infty}$ control design for both these cases. In the meantime reachability [24] and controllability [25] of switched singular systems has been solved, which paved the way for the synthesis of more effective control designs.

This paper focuses on solving for quadratic stability [12] of switched singular linear systems. In contrast to the other methods pointed above out, we deal with the switched singular systems directly and without any transformation. Convex combination technique for standard switched systems is extended to singular linear switched systems in order to derive a sufficient condition of quadratic stability using a stabilizing switching law design. For a switched singular linear system with two subsystems, a necessary and sufficient condition of quadratic stability is obtained. In addition, some initial discussion is provided on the issue of realizing the switching law so as to avoid zeno behavior.

Further this paper is organized as follows. In the next Section II some of the important preliminaries are given. Thereafter in Section III the problem of quadratic stability for the singular linear switched systems is solved. Section IV presents the elaborated procedure for designing the switching law for quadratic stabilization of singular linear switched systems. Section V presents an illustrative example along with the respective numerical results to demonstrate the achieved quadratic stability. Conclusions and references follow thereafter.

\section{PRELIMINARIES}

Consider the singular linear system

$$
E \dot{x}(t)=A x(t), \quad x(0)=x_{0},
$$

where $x \in R^{n}$ is the state vector, $E \in R^{n \times n}, A \in R^{n \times n}$ are constant matrices, and $\operatorname{rank}(E)<n$. If there exists a constant complex number scalar $s \in C$ such that

$$
\operatorname{det}(s E-A) \neq 0 \text {, }
$$

then system (1) is said to be regular. The singular systems that are regular do possess a unique solution. It is this class of singular systems that make up the topic of investigation in this paper. Furthermore, in here all singular systems are assumed to be regular and satisfying the above necessary condition.
When system (1) is regular and $x_{0}$ is a consistent initial condition, then the system has a unique and continuous solution denoted by $x\left(t ; x_{0}, 0\right)$. For the sake of simplicity, we denote the system (1) as pair $(E, A)$.

Definition 1. The set of all consistent initial conditions of system (1) is called consistent initial condition set (CISS), denoted by $D$.

Proposition 1 [16]. Let $x\left(t ; x_{0}, 0\right)$ be a solution of system (1) satisfying $x(0)=x_{0}$. If $x_{0} \in D$, then $x\left(t ; x_{0}, 0\right) \in D$, for any $t>0$.

From Proposition 1 we know that the solution of the singular system (1) entirely lies in $D$ if the initial conditions belong to $D$. This property will be used to ensure the existence of the solution of a switched singular system by imposing some constraints on CISS.

Sometimes, singular system (1) may have impulse solutions which are not solutions in the sense of differential equation. In order to rule out impulse terms, we will need the following lemma.

Lemma 1 [10]. Singular system (1) is regular, stable and impulse free if and only if for any given symmetric positive definite matrix $W>0$, there exists a matrix $P$ satisfying Lyapunov equation:

$$
A^{\mathrm{T}} P+P^{\mathrm{T}} A=-W,
$$

and $E^{\mathrm{T}} P=P^{\mathrm{T}} E \geq 0$.

A singular linear system is said to be admissible if it is regular, stable and impulse free.

\section{QUADRATIC STABILITY FOR SWITCHED SINGULAR LINEAR SYSTEMS}

Consider the following switched linear singular system:

$$
\begin{aligned}
E \dot{x}(t) & =A_{\sigma(t)} x(t), \quad x_{0}=x\left(t_{0}\right), \\
i & \in \Lambda=\{1,2, \cdots, m\},
\end{aligned}
$$

where $x(t) \in R^{n}$ is the state of the system, $E, A_{i} \in R^{n \times n}$ are constant matrices, and the switching law $\sigma(x(t))$ : $R^{n} \rightarrow\{1,2, \cdots, m\}$ is a piecewise constant function.

Let the switching sequence be as follows

$$
\pi=x_{0},\left(t_{0}, i_{0}\right), \cdots,\left(t_{M}, i_{M}\right), i_{0}, \cdots, i_{M} \in \Lambda,
$$

where $x_{0}=x\left(t_{0}\right)$ is the initial condition, the time sequence $\left\{t_{j}\right\}$ satisfies $t_{1}<t_{2}<\cdots<t_{M}$. The switching sequence is called finite if $t_{M}<\infty$; if otherwise it is called infinite.

Definition 2 [16]. We call $x\left(t ; x_{0}, t_{0}\right)$ a solution of 
switched singular system (3), if $E \dot{x}(t)=A_{i_{k}} x(t)$ when $t_{k} \leq t<t_{k+1}$, and $x\left(t_{k}^{-} ; x_{0}, t_{0}\right)=x\left(t_{k}^{+} ; x_{0}, t_{0}\right)$.

It is obvious that the solution is continuous.

Remark 1. If the initial condition $x_{0}$ belongs to a CISS of a subsystem, the trajectory of the system evolves within this CISS when this subsystem is activated. From Proposition 1 and Definition 2, when switching takes place, the state of the "switched off" subsystem must belong to the CISS of the "switched on" subsystem.

This desired feature can be guaranteed [11] by either of the following conditions:

(i) There exists a common decomposition, i.e., there exist two non-singular matrices $Q$ and $P \in R^{n \times n}$ such that

$$
Q E P=\left[\begin{array}{cc}
I_{r} & 0 \\
0 & N
\end{array}\right], Q A_{i} P=\left[\begin{array}{cc}
A_{i 1} & 0 \\
0 & I_{n-r}
\end{array}\right], \quad i \in\{1,2, \cdots, m\}
$$

or

(ii) $D_{i}=D_{j} \forall i, j \in\{1,2, \cdots, m\}$ where

$D_{i}=\left\{x_{0}: x_{0} \in \aleph\left(\mathrm{I}-\hat{E}_{i} \hat{E}_{i}^{D}\right)\right\}, \hat{E}_{i}=\left(\mathrm{s} E-A_{i}\right)^{-1} E$,

and $S$ is any complex scalar.

The null space (.) of a matrix is denoted by $\aleph($.$) ; and above$ the superscript "D" stands for Drazin inverse [11].

Definition 3. If there exist some constant $\varepsilon>0$, switching law $\sigma(x(t))$ and a piecewise continuously differentiable quadratic function $V: R^{n} \rightarrow R^{+}$, with $V(0)=0, V(x)>0$, for $x \neq 0$, and the derivative of $V(x)$ along solution of the system (3) satisfies

$$
\frac{\partial V}{\partial x} A_{i_{k}} x<-\varepsilon\|x\|^{2}
$$

then switched system (3) is said to be quadratically stable.

Definition 4. The system

$$
E \dot{x}(t)=A_{\alpha} x(t)
$$

is called a convex combination system of system (3), where

$$
A_{\alpha}=\sum_{i=1}^{m} \alpha_{\mathrm{i}} A_{i}, \alpha_{\mathrm{i}} \in[0,1] \text { and } \sum_{i=1}^{m} \alpha_{\mathrm{i}}=1
$$

and not all $\alpha_{\mathrm{i}}=0$.

We assume that each subsystem of the system (3) is unstable. Because it is trivial to design a switching law to quadratically stabilize switched singular systems if some or all subsystems are stable. Thus we do not consider cases $\alpha_{\mathrm{i}}=0$ or $\alpha_{\mathrm{i}}=1$.

Theorem 1 Suppose that there exists an admissible convex combination system (4); then there exists a switching law $\sigma(x(t))$ such that makes the singular system
(3) quadratically stable.

Proof: Since system (4) is admissible, from Lemma 1, there exists a matrix $P$ such that

$$
A_{\alpha}^{\mathrm{T}} P+P^{\mathrm{T}} A_{\alpha}<0
$$

and

$$
E^{\mathrm{T}} P=P^{\mathrm{T}} E \geq 0
$$

that is

$$
\sum_{i=1}^{m} \alpha_{i}\left(A_{i}^{\mathrm{T}} P+P^{\mathrm{T}} A_{i}\right)<0
$$

Then for any $x \neq 0$, there exists some $A_{i}, i \in \Lambda$, such that

$$
\left(A_{i}^{\mathrm{T}} P+P^{\mathrm{T}} A_{i}\right)<0 \text {. }
$$

Let $\Omega_{i}^{+}=\left\{x: x^{T} W_{i} x>0, x \neq 0\right\}, \widetilde{D}=\bigcap_{i=1}^{m} D_{i}$, and $W_{i}=-\left(A_{i}^{\mathrm{T}} P+P^{\mathrm{T}} A_{i}\right)$, where $D_{i}$ is the consistent initial condition set of the $i$ th subsystem. In order to guarantee the existence and uniqueness of the solution, we assume $\widetilde{D} \neq \Phi$ ( $\Phi$ is the empty set).

To avoid zeno behavior on the boundaries of the regions, we pick new open conic regions $\widetilde{\Omega}_{i}^{+}, i=1,2, \cdots, m$, and we still have $\bigcup_{i=1}^{m} \widetilde{\Omega}_{i}^{+}=R^{n} \backslash\{0\}$. Naturally, since we investigate the case of the class (3) with unstable subsystems, the state space has to be entirely covered by means of these regions.

Notice that the number

$$
\varepsilon_{i}=\min _{x \in c l \Omega_{i}^{+},\|x\|=1} x^{\mathrm{T}} W_{i} x
$$

is well defined and positive for each $i \in \Lambda$, where "cl" denotes the closure of a given set, accordingly. By choosing a positive number $\varepsilon<\min \left\{\varepsilon_{1}, \varepsilon_{2}, \cdots, \varepsilon_{m}\right\}$, we obtain

$$
x^{\mathrm{T}} W_{i} x>\varepsilon\|x\|^{2}, \text { for any } x \in \widetilde{\Omega}_{i}^{+}, i \in \Lambda .
$$

Now, for any $x_{0} \in \widetilde{D}$, design the switching law as follows:

$$
\begin{gathered}
\text { If } x(t) \in \widetilde{\Omega}_{i}^{+} \text {, then } \sigma(x(t))=i ; \\
\text { If } x(t) \in \bigcap_{k=1}^{l} \widetilde{\Omega}_{i_{k}}^{+}\left(i_{k} \in \Lambda, l \leq m\right), \text { then } \\
\sigma(x(t))=\arg \min _{i}\left\{x^{\mathrm{T}}(t) W_{i} x(t)\right\} .
\end{gathered}
$$

It is easy to verify that this switching law indeed stabilizes system (3). In fact, let choose Lyapunov function candidate as $V(E x)=x^{\mathrm{T}} E^{\mathrm{T}} P x$. Then when $\sigma(x(t))=i$, the time-derivative of $V(E x)$ along the trajectory of the 
system satisfies:

$$
\begin{gathered}
\frac{d V(E x)}{d t}=\dot{x}^{\mathrm{T}} E^{\mathrm{T}} P x+x^{\mathrm{T}} E^{\mathrm{T}} P \dot{x} \\
=x^{\mathrm{T}}\left(A_{i}^{\mathrm{T}} P+P^{\mathrm{T}} A_{i}\right) x \\
=-x^{\mathrm{T}} W_{i} x<-\varepsilon\|x\|^{2} .
\end{gathered}
$$

We have shown that the existence of an admissible convex combination system (4) is a sufficient condition for quadratic stability. Next, we will show that this condition is also necessary if the system (3) has two subsystems.

Consider the following system:

$$
\begin{aligned}
& E \dot{x}(t)=A_{1} x(t) \\
& E \dot{x}(t)=A_{2} x(t)
\end{aligned}
$$

We need the celebrated method of S-procedure.

Lemma 2 [26] (S-procedure) Let $T_{0}, T_{1}$ be $n \times n$ symmetric matrices. If for any $x \neq 0$ which satisfies $x^{\mathrm{T}} T_{1} x \geq 0$, the inequality $x^{\mathrm{T}} T_{0} x>0$ is true, and there is some $x_{0}$ satisfying $x_{0}^{\mathrm{T}} T_{1} x_{0}>0$, then there exists a scalar $\beta \geq 0$ such that $T_{0}-\beta T_{1}>0$.

Now we are in the position to present the other main result of this study.

Theorem 2 Switched singular system (8) is quadratically stable if and only if there exists $\alpha \in(0,1)$ such that system $\left(E, A_{\alpha}\right)$ is admissible, where $A_{\alpha}=\alpha A_{1}+(1-\alpha) A_{2}$.

Proof: Apparently the sufficiency follows from the Theorem 1 . Hence the necessity only is proved in the sequel.

Assume the switching law $\sigma(x(t))$ does quadratically stabilize the system (8). Then for any $x \neq 0$, there exists $\varepsilon>0$ and matrix $P$, such that either

$$
x^{\mathrm{T}}\left(A_{1}^{\mathrm{T}} P+P^{\mathrm{T}} A_{1}\right) x<-\varepsilon x^{\mathrm{T}} x,
$$

or

$$
x^{T}\left(A_{2}^{\mathrm{T}} P+P^{\mathrm{T}} A_{2}\right) x<-\varepsilon x^{\mathrm{T}} x,
$$

or, equivalently,

$$
\begin{aligned}
& \text { if } x^{\mathrm{T}}\left(A_{2}^{\mathrm{T}} P+P^{\mathrm{T}} A_{2}+\varepsilon I\right) x \geq 0 \text {, then } \\
& x^{\mathrm{T}}\left(-A_{1}^{\mathrm{T}} P-P^{\mathrm{T}} A_{1}-\varepsilon I\right) x>0, \\
& \text { else if } x^{\mathrm{T}}\left(A_{1}^{\mathrm{T}} P+P^{\mathrm{T}} A_{1}+\varepsilon I\right) x \geq 0 \text {, then } \\
& x^{\mathrm{T}}\left(-A_{2}^{\mathrm{T}} P-P^{\mathrm{T}} A_{2}-\varepsilon I\right) x>0 .
\end{aligned}
$$

It is not difficult to prove that if for any $x \neq 0$, $x^{\mathrm{T}}\left(A_{2}^{\mathrm{T}} P+P^{\mathrm{T}} A_{2}+\varepsilon I\right) x \geq 0$ is satisfied the system (8-a) is stable, and if for any $x \neq 0$,
$x^{\mathrm{T}}\left(A_{1}^{\mathrm{T}} P+P^{\mathrm{T}} A_{1}+\varepsilon I\right) x \geq 0$ is satisfied the system (8-b) is stable. Without loss of generality, in the following discussion we discard the trivial cases above.

By virtue of the S-procedure, we know that there exists some $\beta>0$ such that

$x^{\mathrm{T}}\left(A_{1}^{\mathrm{T}} P+P^{\mathrm{T}} A_{1}+\beta\left(A_{2}^{\mathrm{T}} P+P^{\mathrm{T}} A_{2}\right)\right) x<-(1+\beta) \varepsilon x^{\mathrm{T}} x$ or, equivalently,

$$
x^{\mathrm{T}}\left(P^{\mathrm{T}} \frac{A_{1}+\beta A_{2}}{1+\beta}+\frac{A_{1}^{\mathrm{T}}+\beta A_{2}^{\mathrm{T}}}{1+\beta} P\right) x<-\varepsilon x^{\mathrm{T}} x .
$$

Therefore, the matrix $A_{\alpha}=\left(A_{1}+\beta A_{2}\right) /(1+\beta)$ is convex combination of $A_{1}, A_{2}$, and system $\left(E, A_{\alpha}\right)$ is quadratically stable; in there $\alpha=1 /(1+\beta) \in(0,1)$.

Remark 2. From the proof of Theorem 1 and Theorem 2, we can see that the switching law for the singular switched system depends on the system matrix of the respective convex combination system. If the directions of vector fields of subsystems are different from each other, the designed switching law may result in sliding modes or fast switching. These are unacceptable phenomena in practical applications. It is therefore that further investigations discussion on how to avoid zeno phenomena is needed, which remains an open issue for future research. Here a modest discussion on this issue is given.

In the sequel, for the sake of simplicity, only the case of two subsystems is investigated further.

\section{A Design Of Quadratically STABilizing SWITCHING LAW}

Since system $\left(E, A_{\alpha}\right)$ is admissible, from lemma 1 we know that there exists a matrix $P$ such that

$$
A_{\alpha}^{\mathrm{T}} P+P^{\mathrm{T}} A_{\alpha}<0 \text { and } E^{\mathrm{T}} P=P^{\mathrm{T}} E \geq 0 .
$$

It follows that

$$
\alpha\left(A_{1}^{\mathrm{T}} P+P^{\mathrm{T}} A_{1}\right)+(1-\alpha)\left(A_{2}^{\mathrm{T}} P+P^{\mathrm{T}} A_{2}\right)<0 .
$$

For $\quad$ any $\quad x \in R^{n} \backslash\{0\} \quad, \quad \alpha\left(A_{1}^{\mathrm{T}} P+P^{\mathrm{T}} A_{1}\right)<0 \quad$ or $(1-\alpha)\left(A_{2}^{\mathrm{T}} P+P^{\mathrm{T}} A_{2}\right)<0$ is true. Let define

$$
\begin{gathered}
W_{i}=-\left(A_{i}^{\mathrm{T}} P+P^{\mathrm{T}} A_{i}\right) \quad \mathrm{i}=1,2 \\
\Omega_{i}^{+}=\left\{x: x^{\mathrm{T}} W_{i} x>0, x \neq 0\right\} \\
\Omega_{i}^{0}=\left\{x: x^{\mathrm{T}} W_{i} x=0, x \neq 0\right\}
\end{gathered}
$$

Apparently, neither matrix $W_{1}$ nor $W_{2}$ is not definite. In fact, if $W_{1}$ is positive definite, then system (8a) is stable, 
which contradicts Assumption 1. Similarly, we know that $W_{2}$ is not positive definite. We also know there exists $x \in R^{n}, x \neq 0$, which belongs to $\Omega_{1}^{+} \cap \Omega_{2}^{+}$.

Now, we define the following functions used to determine switching surfaces

$$
\begin{aligned}
& S_{1}(x)=x^{\mathrm{T}}\left(W_{1}-\gamma W_{2}\right) x, \\
& S_{2}(x)=x^{\mathrm{T}}\left(W_{2}-\gamma W_{1}\right) x,
\end{aligned}
$$

where $0<\gamma<1 . S_{1}(x), S_{2}(x)$ satisfy the following properties:

(1) $S_{1}(x) \geq 0, S_{2}(x) \geq 0 \quad, \quad x \neq 0 \quad$ implies $x \in \Omega_{1}^{+} \cap \Omega_{2}^{+}$;

(2) $S_{1}(x)$ and $S_{2}(x)$ cannot simultaneously be non-positive if $x \neq 0$;

(3) $x \neq 0$ on the boundary of $\Omega_{i}^{+}$implies $S_{i}(x)<0$ and $S_{j}(x)>0$.

On the grounds of the above properties of $S_{1}(x)$ and $S_{2}(x)$, the switching law $\sigma(x(t))$ is designed as follows:

1) for any $x_{0} \in D_{1} \cap D_{2}$, if $x_{0} \in \Omega_{1}^{+}$then $\sigma\left(x\left(t_{0}\right)\right)=1$, otherwise $\sigma\left(x\left(t_{0}\right)\right)=2$;

2) if $\sigma\left(x\left(t^{-}\right)\right)=1, x \in \Omega_{1}^{+} \cap \Omega_{2}^{+}$and $S_{1}(x) \leq 0$, then $\sigma\left(x\left(t^{+}\right)\right)=2$;

3) if $\sigma\left(x\left(t^{-}\right)\right)=2, x \in \Omega_{1}^{+} \cap \Omega_{2}^{+}$and $S_{2}(x) \leq 0$, then $\sigma\left(x\left(t^{+}\right)\right)=1$.

From the definition of switching law $\sigma(x(t))$ we have $x\left(t ; x_{0}, t_{0}\right) \in D_{1} \cap D_{2}$ for any $x_{0} \neq 0, x_{0} \in D_{1} \cap D_{2}$ as shown in [12]. If $x_{0} \in \Omega_{i}^{+}$for $i=1,2$, then the $i$ th subsystem is activated and $S_{i}(x)>0$. So we have $x \in \Gamma=\Omega_{i}^{+} \bigcap\left\{x: S_{i}(x)>0\right\}$. Now we consider $\widetilde{\Gamma}$, the closure of the set $\Gamma$. It is easy to know that the boundary of $\Omega_{i}^{+}$is not contained in $\widetilde{\Gamma}$. This is because if $x \neq 0$ and $x \in \Omega_{i}^{0}$, then $S_{i}(x)<0$. The function $x^{\mathrm{T}} W_{i} x$ achieves its minimum over the set $\widetilde{\Gamma}$ at some $\bar{x}$ when restricted to $\|x\|=1$.

Further next, denote the minimum $\eta_{i}$, i.e. $\bar{x}^{\mathrm{T}} W_{i} \bar{x}=\eta_{i}$. Then for any $x \in \Omega_{i}^{+} \cap\left\{x: S_{i}(x)>0\right\}$ we have $x^{\mathrm{T}}\left(A_{i}^{\mathrm{T}} P+P^{\mathrm{T}} A_{i}\right) x \leq-\eta_{i} x^{\mathrm{T}} x$ hence the system $(8)$ is quadratically stable by means of the designed switching law.

\section{ILLUSTRATIVE EXAMPLE}

To illustrate the just derived results, we consider the switched singular system as given below. Namely, we consider

$$
E \dot{x}(t)=A_{\sigma(x(t))} x(t)
$$

with

$$
x=\left[x_{1}, x_{2}, x_{3}, x_{4}\right]^{\mathrm{T}}, \sigma(x(t)) \in\{1,2\},
$$

and

$$
\begin{gathered}
E=\left[\begin{array}{cccc}
1 & 0 & 1 & 0 \\
0 & 1 & 0 & 0 \\
0 & 0 & 0 & 0 \\
0 & -1 & 0 & 0
\end{array}\right], A_{1}=\left[\begin{array}{cccc}
2 & 0 & 1.6667 & 0 \\
0 & -4 & -0.6667 & 0 \\
0 & 0 & 0.3333 & 0 \\
-1 & 2 & -0.3333 & 1
\end{array}\right], \\
A_{2}=\left[\begin{array}{cccc}
-4 & 0 & -4.3333 & 0 \\
0 & 0 & -0.6667 & 0 \\
0 & 0 & 0 & 0 \\
-1 & -2 & -0.3333 & 1
\end{array}\right] .
\end{gathered}
$$

It is obvious that both systems $\left(E, A_{1}\right)$ and $\left(E, A_{2}\right)$ are unstable since their finite poles are found to be $2,-4$ and 0 , -4 , respectively.

Now let consider $A_{\alpha}=\alpha A_{1}+(1-\alpha) A_{2}$. When $\alpha=0.5$, there exists a matrix as given below

$$
P=\left[\begin{array}{cccc}
1 & 0.6667 & 1 & 0 \\
0.1667 & 0.25 & 0.1667 & -0.5 \\
5.3333 & 3.1667 & 2.3333 & 0 \\
-0.5 & -1 & -05 & -0.5
\end{array}\right]
$$

such that

$$
E^{\mathrm{T}} P=P^{\mathrm{T}} E=\left[\begin{array}{cccc}
1 & 0.6667 & 1 & 0 \\
0.6667 & 1.25 & 0.6667 & 0 \\
1 & 0.6667 & 1 & 0 \\
0 & 0 & 0 & 0
\end{array}\right] \geq 0
$$

and also

$$
A_{\alpha}^{\mathrm{T}} P+P^{\mathrm{T}} A_{\alpha}=-I<0 .
$$

Thus it follows from Theorem 1 that the switched singular system is quadratically stable.

\section{CONCLUSION}

The convex combination technique has been extended to switched singular systems to give a sufficient condition for quadratic stability. In addition, for systems with only two subsystems this condition is shown to be also the necessary one. A stabilizing switching law has been designed and some discussion on avoiding zeno phenomena was given too. 
It should be noted unlike in conventional switched linear systems where the convex combination technique is fully understood, the case with switched singular systems is more involved. Thus we had to overcome considerable difficulties to make the convex combination technique applicable to switched singular systems.

Furthermore, it should be also noted the above resented novel results, given the solely switching law employed, compare favorably with both the design results by $\mathrm{Fu}$ and co-authors in [16-18] and by Gao-Wu [19], respectively, where considerably more sophisticated control strategies are proposed. For the simplicity of switching law is rather appealing for practical applications

Finally, as it may well be inferred from the recent article by $\mathrm{Wu}$ and Liberzon [27], the underlying essentialities the considered problem for singular linear switched systems are also related to the invertibility problem.

Yet, it can be argued that a new and promising approach would be the potentially feasible generalization of multiple Lyapunov function method to switched singular systems. As far as the forms of systems are concerned, the study of a more general switched singular system with $E_{i} \neq E_{j}$ may be much more challenging.

\section{ACKNOWLEDGEMENT}

The authors express their profound gratitude to those anonymous reviewers who provided constructive remarks and suggestions in their respective reviews, which helped improve the presentation quality of this contributed paper.

\section{REFERENCES}

[1] M. S. Branicky, "Stability of switched and hybrid systems," in Proceedings of the $33^{r d}$ Conference on Decision and Control, Lake Buena Vista, FL, 1994, pp. 3498-3503.

[2] R. A. DeCarlo, M. S. Branicky, S. Petterson, and B. Lennartson, "Perspective and results on the stability and stabilizability of hybrid systems," in Proceedings of the IEEE, vol. 88, no. 7, pp.1069-1082, July 2000.

[3] P. Peleties, R. A. DeCarlo, "Asymptotic stability of m-switched systems using Lyapunov-like function," in Proceedings of the $11^{\text {th }}$ American Control Conference, 1992, pp. 1679-1684.

[4] M. A. Wicks, P. Peleties, and R. A. DeCarlo, "Construction of piecewise Lyapunov function for stabilizing switched systems," in Proceedings of the $33^{\text {rd }}$ Conference on Decision and Control, Lake (M. K.Macmasten, General Chair, and H. H. McClamrosh, Program Chair), Buena Vista, FL, December 14-16. The IEEE, Piscatway, NJ, 1994, pp. 3492-3497.

[5] E. Feron, Quadratic Stabilizability of Switched Systems via State and Output Feedback, Technical report CICS-P-468, The MIT, 1996.

[6] D. Liberzon and A S. Morse, "Basic problems in stability and design of switched systems," IEEE Control Systems Magazine, vol. 19, no. 5, pp. 59-70, 1999.

[7] D. Liberzon, Switching in Systems and Control. Boston, MA: Birkhauser, 2003.

[8] Z. Sun, S. S. Ge, Switched Linear Systems - Control and Design. London: Springer, 2005.

[9] L. Dai, Singular Control Systems. Berlin: Springer-Verlag, 1989, pp. 1-101.
[10] Q. L. Zhang, D. M. Yang, Analysis and Synthesis for Uncertain Descriptor Systems. Shenyang: NEU Press, 2003, Chapter 3.

[11] S. L. Campbell, Singular Systems of Differential Equations. Marshfields, MA: Pitmax, 1980.

[12] J. Zhao and G. M. Dimirovski, "Quadratic stability of nonlinear switched systems," IEEE Transactions on Automatic Control, vol. 49, no. 4, pp. 574-578, 2004.

[13] M. A. Wicks, P. Peleties, and R. A. DeCarlo, "Switched controller synthesis for the quadratic stabilization of a pair of unstable linear systems," European Journal of Control, vol. 4, pp. 140-147, 1998.

[14] S. Ibrir, E. K. Boukas, "Stabilization of singular switching systems with constant gain controllers," International Journal of Hybrid Systems, vol. 3, pp. 151-164, 2003.

[15] B. Men, J. F. Zhang, "Admissible switched control of singular systems," in Proceedings of the $23^{\text {rd }}$ Chinese Control Conference, Shanghai, P.R. China, 2004, pp. 1615-1619.

[16] Z.-M. Fu, S.-M. Fei, "Robust $H_{\infty}$ dynamic output feedback stabilization for a class of uncertain switched singular systems." Acta Automatica Sinica, vol. 34, no. 4, pp. 482-487, 2008.

[17] Z.-M. Fu, S.-M. Fei, " $H_{\infty}$ control for a class of switched linear singular systems." Control Theory \& Applications, vol. 25, no. 4, pp. 693-698, 2008

[18] Z.-M. Fu, S.-M. Fei, Y.-M. Bo, T. Li, "Robust $H_{\infty}$ control for a class of switched uncertain singular systems." Journal of Nanjing University of Science \& Technology, vol. 32, no. 3, pp. 269-273, 2008.

[19] L. Gao, Y. Wu, "An LMI-based variable structure control for a class of uncertain singular Markov switched systems." Journal of Control Theory \& Applications, vol. 5, no. 4, pp. 415-419, 2007.

[20] Y. J. Yin, Y. Z. Liu, and J. Zhao, "The existence and uniqueness of solution for switched linear singular systems", in Proceedings of the $17^{\text {th }}$ Chinese Control and Decision Conference, Harbin, P.R. China, 2005, pp. 105-108.

[21] Y.-J. Yin, Y.-Z. Liu, J. Zhao, "Stability of a class of switched linear singular systems." Control \& Decision, vol. 21, no. 1, pp. 24-27, 2006.

[22] Y.-J. Yin, Y.-Z. Liu, J. Zhao, "Stability of a class of switched linear singular systems with impulsive effects." Acta Automatica Sinica, vol. 33, no. 4, pp. 446-448, 2007.

[23] Y. Yin, J. Zhao, Y. Liu, "H-infinity control of switched and impulsive singular systems." Journal of Control Theory \&Applications, vol. 6, no.1, pp. 86-92, 2008.

[24] B. Meng, J.F. Zhang, "Reachability conditions for switched linear singular systems." IEEE Transaction on Automatic Control, vol. 51, no. 3, pp. 482-488, 2006

[25] B. Meng, J.F. Zhang, "Controllability conditions for switched linear singular systems." Dynamics of Continuous, Discrete \& Impulsive Systems Series B: Applications \& Algorithms, vol. 14, no. 5, pp. 641-657, 2007

[26] S. Boyd, L. E. Ghaoui, E. Feron, and V. Balakrishnan, Linear Matrix Inequalities in Systems and Control Theory, SIAM Studies in Applied Mathematics, vol. 15, Philadelphia, PA, 1994, Chapter 2.

[27] L. Vu and D. Liberzon, "Invertibility of switched linear systems." Automatica, vol. 44, no. 4, pp. 949-958, 2008. 